\title{
Searching for rare FCNC decays at BESIII
}

\author{
Dayong Wang ${ }^{* \dagger}$ \\ for the BESIII Collaboration
}

State Key Laboratory of Nuclear Physics and Technology, Peking University, Beijing, China

E-mail: dayong • wang@pku.edu.cn

BESIII at BEPCII collider is a tau-charm factory with huge samples of on threshold charm meson pairs, directly produced charmonia and many other unique data sets. The Flavor Changing Neutral Current decays(FCNC) are forbidden at tree level in the Standard Model (SM) and are thus rare. Any direct observation beyond SM expectations could be a good probe of physics beyond SM. BESIII could probe these FCNC decays from multiple channels. Here we review some of search results of FCNC decays from BESIII. We present the latest results of searches for the decay of $J / \psi \rightarrow D^{0} e^{+} e^{-}, \psi(3686) \rightarrow D^{0} e^{+} e^{-}, \psi(3686) \rightarrow \Lambda_{c}^{+} \bar{p} e^{+} e^{-}, D \rightarrow h\left(h^{\prime}\right) e^{+} e^{-}$. More results are coming in the future.

XXIX International Symposium on Lepton Photon Interactions at High Energies - LeptonPhoton2019 August 5-10, 2019

Toronto, Canada

\footnotetext{
* Speaker.

${ }^{\dagger}$ Partially supported by Joint Funds of the National Natural Science Foundation of China (Grants No. U1832207) and by Ministry of Science and Technology(Grants No. 2015CB856700).
} 


\section{Introduction}

The Flavor Changing Neutral Current decays(FCNC) are forbidden at tree level in the Standard Model (SM) due to the Glashow-Iliopoulos-Maiani mechanism [1], and are thus rare. They could only contribute through loops. Any direct observation beyond SM expectations could be a good probe of physics beyond SM.

BEPCII is a $\tau$-charm factory running at the c. m. energy range of $2.0-4.6 \mathrm{GeV}$, located at suburbs of Beijing. The unique features in this energy range allow a rich physics program. The BESIII is a hermetic detector with geometrical acceptance of $93 \%$ of $4 \pi$ and consists of a smallcelled, helium-based draft chamber, a $\mathrm{CsI}(\mathrm{Tl})$ crystal electromagnetic calorimeter, a time-of-flight system and a muon chamber system incorporated in the return yoke of 1T solenoid. More details of the detector are described in Ref [2].

The BESIII experiment has accumulated world largest data sets of 10 billion $J / \psi$ 's, 0.5 billion $\psi(3686)$ 's and $2.9 \mathrm{fb}^{-1}$ at $\psi(3773)$ that are directly produced. With these large data sets, together with the clean experimental environment and high luminosity, BESIII is ideally posed for searches for new physics beyond standard model. BESIII has performed several such FCNC decays, which are summarized in this paper. Charge conjugation is always implied throughout.

\section{Search for $J / \psi \rightarrow D^{0} e^{+} e^{-}$and $\psi(3686) \rightarrow D^{0} e^{+} e^{-}$[3]}

In the SM, the decay branching fraction for this kind of rare process is expected to be of order $10^{-10}$ to $10^{-13}$. Some new physics models could have orders of magnitude higher decay rates, which could in the reach of BESIII.
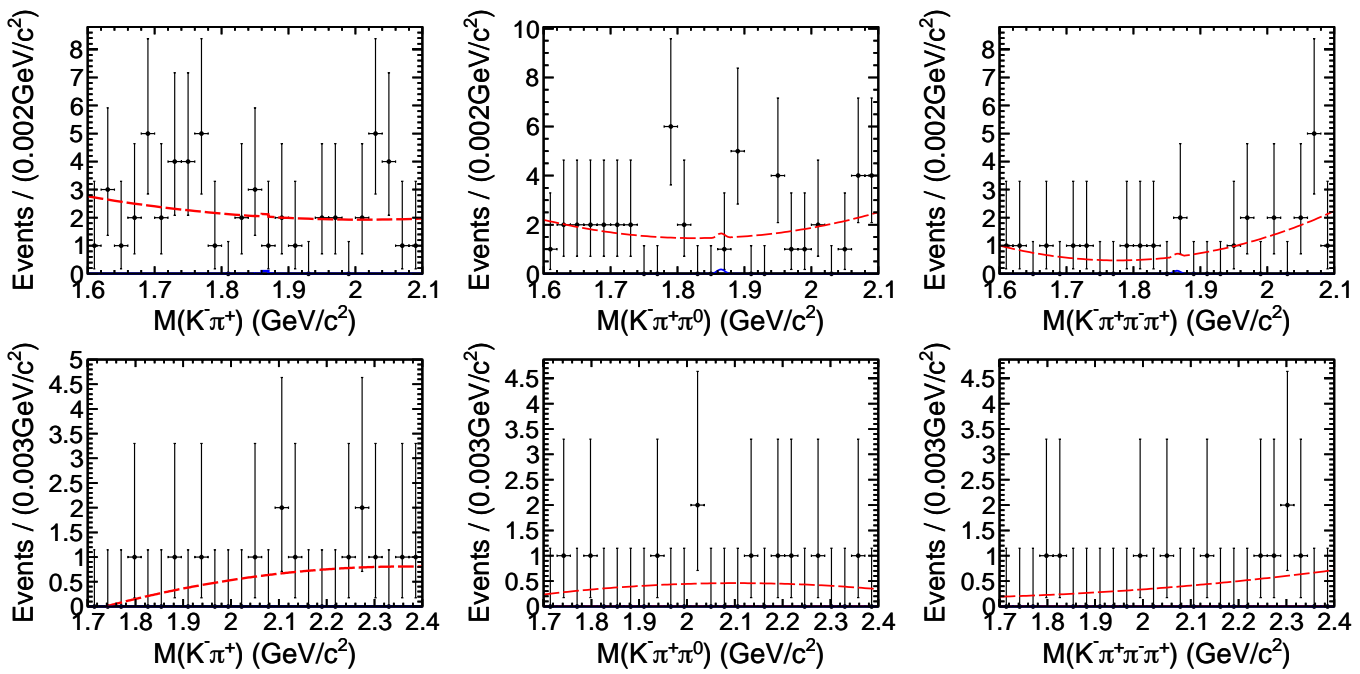

Figure 1: Distributions of $K^{-} \pi^{+}$(left column), $K^{-} \pi^{+} \pi^{0}$ (middle column) and $K^{-} \pi^{+} \pi^{-} \pi^{+}$(right column) invariant masses. The top and bottom rows are for the $J / \psi$ and $\psi(3686)$ samples, respectively. Dots with error bars are data, the solid and dashed curves are for the signal shape and the total best fit to data, respectively.

This search is performed with $(1310.6 \pm 7.2) \times 10^{6} \mathrm{~J} / \psi$ events and $(448.1 \pm 2.9) \times 10^{6}$ $\psi(3686)$ events, collected by BESIII. The $D^{0}$ signals are reconstructed through its three prominent 
exclusive hadronic decay modes, $K^{-} \pi^{+}, K^{-} \pi^{+} \pi^{0}$, and $K^{-} \pi^{+} \pi^{-} \pi^{+}$, which have relatively large branching fractions, and relatively low background. After requring one $D^{0}$ and one pair of $e^{+} e^{-}$ reconstructed, the distributions of the invariant masses from the three $D^{0}$ meson decay modes are simultaneously fit with unbinned maximum likelihood method for the $J / \psi$ and $\psi(3686)$ samples. The distributions together with the best fit curves are shown in Fig. 1.

No $D^{0}$ signals are observed, and we compute the upper limits(UL) on the branching fraction at the $90 \%$ C.L. using a Bayesian method [4] with a flat prior,the correlated and un-correlated systematic uncertainties are incorporated. The results are $\mathscr{B}\left(J / \psi \rightarrow D^{0} e^{+} e^{-}\right)<8.5 \times 10^{-8}$ and $\mathscr{B}\left(\psi(3686) \rightarrow D^{0} e^{+} e^{-}\right)<1.4 \times 10^{-7}$, respectively. The limit on $\mathscr{B}\left(J / \psi \rightarrow D^{0} e^{+} e^{-}\right)$is more stringent by two orders in magnitude compared to the previous results, and the $\mathscr{B}(\psi(3686) \rightarrow$ $\left.D^{0} e^{+} e^{-}\right)$is set for the first time.

\section{Search for $\psi(3686) \rightarrow \Lambda_{c}^{+} \bar{p} e^{+} e^{-}[5]$}

The Feynman diagram of this process is shown in Fig. 2(left). The search is performed with $(448.1 \pm 2.9) \times 10^{6} \psi(3686)$ events collected by BESIII. The decay $\psi(3686) \rightarrow \Lambda_{c}^{+} \bar{p} e^{+} e^{-}$with $\Lambda_{c}^{+} \rightarrow p K^{-} \pi^{+}$is reconstructed with six charged tracks with zero net charge. The number of signal events is determined by examining the $\Lambda_{c}^{+}$signal in the $M_{p K^{-} \pi^{+}}$distribution, which is shown in Fig. 2(right).
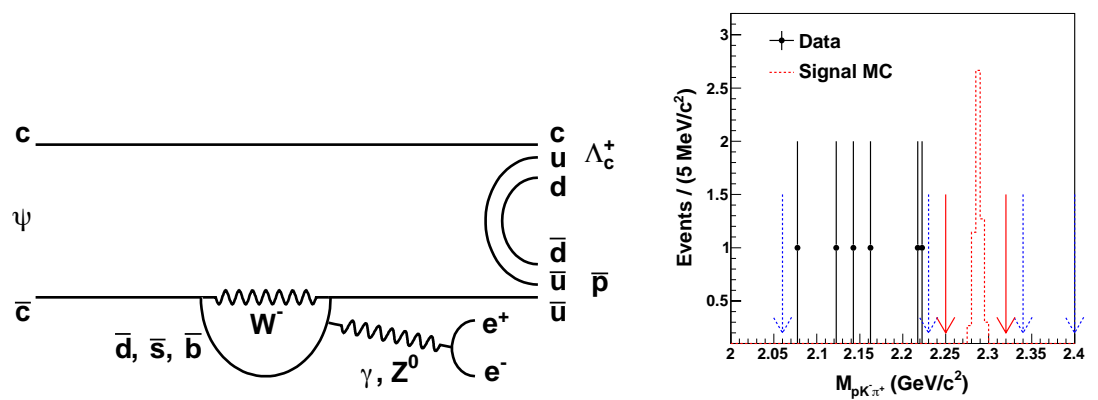

Figure 2: (Left)Feynman diagram for $\psi(3686) \rightarrow \Lambda_{c}^{+} \bar{p} e^{+} e^{-}$. (Right) Distribution of $M_{p K^{-} \pi^{+}}$for the data (dots with error bars) and signal MC (dashed histogram).

No events survive within the signal region ranging from 2.25 to $2.32 \mathrm{GeV} / c^{2}$. The potential background in the signal region is estimated using events in the $M_{p K^{-}} \pi^{+}$sideband regions to be 1.5. No background events are found with the inclusive MC sample and the large data sample of $\sqrt{s}=3.773 \mathrm{GeV}$. As no candidate events are found in the signal region, the estimated number of background events is determined to be $0 \pm 1.5$ events. The upper limit on the BF $(\mathscr{B})$ of the decay $\psi(3686) \rightarrow \Lambda_{c}^{+} \bar{p} e^{+} e^{-}+c . c$. is determined to be $1.7 \times 10^{-6}$. The upper limit is still higher than the SM expectations [6], and no evidence for new physics is found.

\section{Search for $D \rightarrow h\left(h^{\prime}\right) e^{+} e^{-}$[7]}

With the $e^{+} e^{-}$collision sample corresponding to an integrated luminosity of $2.93 \mathrm{fb}^{-1}$ collected at $\sqrt{s}=3.773 \mathrm{GeV}$, BESIII performs a search for the rare decays of $D \rightarrow h\left(h^{\prime}\right) e^{+} e^{-}$, where 
$h^{(\prime)}$ are hadrons. A double tag method is used in the analysis. For each signal mode, $\Delta E_{\text {sig }}$ is required to be within $3 \sigma$ of the nominal value, and only the combination with the smallest $\left|\Delta E_{\text {sig }}\right|$ is kept. No significant excess over the expected backgrounds is observed in $M_{\mathrm{BC}}^{\text {sig }}$ distributions of the surviving events.

The ULs on the signal BFs at the $90 \% \mathrm{CL}$ are determined. The maximal signal significance is $2.6 \sigma$, for $D^{0} \rightarrow K^{-} \pi^{+} e^{+} e^{-}$. Its BF is expected to be dominated by the long distance Bremsstrahlung and (virtual) resonance decay contributions in the lower and upper regions, so we divide the $M_{e^{+}} e^{-}$ distribution into three regions and determine the BFs in the individual regions.

Table 1: Results of the ULs on the BFs for the investigated rare decays of $D \rightarrow h\left(h^{\prime}\right) e^{+} e^{-}$at the $90 \% \mathrm{CL}$, and the corresponding results in the PDG.

\begin{tabular}{lcc||lcc} 
Signal decays & $\mathscr{B}\left(\times 10^{-5}\right)$ & PDG $[4]\left(\times 10^{-5}\right)$ & Signal decays & $\mathscr{B}\left(\times 10^{-5}\right)$ & PDG $[4]\left(\times 10^{-5}\right)$ \\
\hline$D^{+} \rightarrow \pi^{+} \pi^{0} e^{+} e^{-}$ & $<1.4$ & - & $D^{0} \rightarrow K^{-} K^{+} e^{+} e^{-}$ & $<1.1$ & $<31.5$ \\
$D^{+} \rightarrow K^{+} \pi^{0} e^{+} e^{-}$ & $<1.5$ & - & $D^{0} \rightarrow \pi^{+} \pi^{-} e^{+} e^{-}$ & $<0.7$ & $<37.3$ \\
$D^{+} \rightarrow K_{S}^{0} \pi^{+} e^{+} e^{-}$ & $<2.6$ & - & $D^{0} \rightarrow K^{-} \pi^{+} e^{+} e^{\dagger}$ & $<4.1$ & $<38.5$ \\
$D^{+} \rightarrow K_{S}^{0} K^{+} e^{+} e^{-}$ & $<1.1$ & - & $D^{0} \rightarrow \pi^{0} e^{+} e^{-}$ & $<0.4$ & $<4.5$ \\
$\dagger$ in $M_{e^{+}} e^{-}$regions: & & - & $D^{0} \rightarrow \eta e^{+} e^{-}$ & $<0.3$ & $<11$ \\
{$[0.00,0.20) \mathrm{GeV} / c^{2}$} & $<3.0\left(1.5_{-0.9}^{+1.0}\right)$ & - & $D^{0} \rightarrow \omega e^{+} e^{-}$ & $<0.6$ & $<18$ \\
{$[0.20,0.65) \mathrm{GeV} / c^{2}$} & $<0.7$ & $D^{0} \rightarrow K_{S}^{0} e^{+} e^{-}$ & $<1.2$ & $<11$ \\
{$[0.65,0.90] \mathrm{GeV} / c^{2}$} & $<1.9\left(1.0_{-0.4}^{+0.5}\right)$ & - & & & \\
\hline
\end{tabular}

The results are consistent with the SM predictions, as summarized in Table 1. For the four-body $D^{+}$decays, the searches are performed for the first time. The reported ULs of the $D^{0}$ decays are improved in general by a factor of 10, compared to previous measurements [4]. All the measured ULs on the BFs are above the SM predictions, with both long and short distance contributions.

\section{Summary and outlook}

BESIII has performed a series of rare searches for FCNC processes containing transitions of c quark to u quark. This is the only place that the upper quark sector FCNC processes could be probed. Though most of the upper limits are still above than the SM predictions, they may help to discriminate the different new physics models or to constrain the parameters in the different physics models. Additionally, higher statistics $J / \psi, \psi(3686)$ and D meason samples has helped to improve the sensitivity of the measurements. BESIII efforts with more related channels and more coming data will be continued to further test the standard model with higher precision in future.

\section{References}

[1] S. L. Glashow, J. Iliopoulos and L. Maiani, Phys. Rev. D 2, 1285 (1970).

[2] M. Ablikim et al. (The BESIII Collaboration), Nucl. Instrum. Meth. A 614, 345 (2010).

[3] M. Ablikim et al. (The BESIII Collaboration), Phys. Rev. D 96, 111101 (2017).

[4] M. Tanabashiet al. (Particle Data Group), Phys. Rev. D 98, 030001 (2018) and 2019 update.

[5] M. Ablikim et al. (The BESIII Collaboration), Phys. Rev. D 97, 091102 (2018).

[6] M. A. Sanchis-Lonzano, Z. Phys. C 62, 271 (1994); Y. M. Wang et al., Eur. Phys. J. C 54, 107 (2008).

[7] M. Ablikim et al. (BESIII Collaboration), Phys. Rev. D 97, 072015 (2018). 